\title{
Durchführung präparativer Hämapheresen zur Gewinnung von Blutbestandteilkonzentraten - Empfehlungen zur präparativen Hämapherese der Deutschen Gesellschaft für Transfusionsmedizin und Immunhämatologie (DGTI)*
}

\author{
Gert Matthes $^{a} \quad$ Rainer Moog ${ }^{b} \quad$ Hartmut Radtke ${ }^{c} \quad$ Markus Wiesneth $^{d} \quad$ Jürgen Zingsem ${ }^{e}$ \\ a Institut für Transfusionsmedizin, Universitätsklinikum Leipzig, \\ b Institut für Transfusionsmedizin, Universitätsklinikum Essen, \\ c Institut für Transfusionsmedizin, Charité - Universitätsmedizin Berlin, Campus Mitte, Berlin \\ dInstitut für Klinische Transfusionsmedizin und Immungenetik Ulm gGmbH, \\ eTransfusionsmedizinische und Hämostaseologische Abteilung, Universitätsklinikum Erlangen, Deutschland
}

\section{Präambel}

Die «Richtlinien zur Gewinnung von Blut und Blutbestandteilen und zur Anwendung von Blutprodukten (Hämotherapie)» des Wissenschaftlichen Beirats der Bundesärztekammer und des Paul-Ehrlich-Instituts [1] beschreiben im Kapitel 2 «Präparative Hämapherese» die Minimalanforderungen für die Herstellung von Apheresepräparaten.

Die hier vorgelegten «Empfehlungen zu präparativen Hämapheresen» ergänzen diese Richtlinien und geben Durchführungsempfehlungen. Sie lösen die früheren Empfehlungen zur Durchführung apparativer Plasmapheresen [2], präparativer Leuko- und Thrombozytapheresen [3] und Blutstammzellapheresen [4] ab. Diese ausführliche Ergänzung der Richtlinien soll unter Beachtung der Vorgaben für die Herstellung von Blutbestandteilpräparaten [5-10] Erläuterungen und praktische Hilfen für die Hämapherese bieten, damit die größtmögliche Spender- und Patientensicherheit sowie Präparatequalität gewährleistet ist.

\section{Definition der Hämapherese}

Die präparative Hämapherese unterscheidet sich von der konventionellen Vollblutspende durch die Anwendung von Blutzellseparatoren mit extrakorporalem Kreislauf der Spender und Patienten. Sie ermöglicht dadurch die direkte Auftrennung von Blut in verschiedene Bestandteile während des Entnahmevorgangs.

*Vorbereitet durch die Kommission zur Aktualisierung der «Empfehlungen zur präparativen Leuko- und Thrombozytapherese»
Mit Hilfe dieser Zellseparationsverfahren können einzelne Blutbestandteilkonzentrate (Erythrozyten, Thrombozyten, Granulozyten, Lymphozyten, Monozyten, Blutstammzellen oder Plasma) oder gleichzeitig verschiedene Blutkomponenten (Multikomponentengewinnung) entnommen werden. Die nicht benötigten Blutbestandteile werden dabei den Spendern/Patienten direkt wieder zugeführt. Es gibt kontinuierliche und diskontinuierliche Separationsmethoden zur Herstellung von Blutbestandteilpräparaten für die Transfusion und Transplantation. Der extrakorporale Kreislauf und der Einsatz von Zellseparatoren am Menschen stellen eine wesentliche Änderung im Vergleich zur konventionellen Vollblutspende dar und erfordern besondere Sicherheits- und Vorsichtsmaßnahmen, die in diesen Empfehlungen festgelegt sind.

\section{Nomenklatur}

Die präparative Hämapherese ist die Gewinnung autologer oder allogener Blutbestandteilkonzentraten durch gezielte Entnahme bestimmter Komponenten mit Hilfe eines Zellseparators. Sie umfasst:

- Erythrozytapherese $=$ Entnahme von Erythrozyten

- Thrombozytapherese $=$ Entnahme von Thrombozyten

- Granulozytapherese = Entnahme von Granulozyten

- Lymphozytapherese = Entnahme von Lymphozyten

- Monozytapherese $=$ Entnahme von Monozyten

- Blutstammzellapherese $=$ Entnahme von Blutstammzellen

- Plasmapherese = Entnahme von Plasma

- Multikomponentenapherese $=$ Entnahme und gleichzeitige Auftrennung von verschiedenen Blutbestandteilpräparaten.

\begin{tabular}{ll}
\hline KARGER & @ 2007 S. Karger GmbH, Freiburg \\
Fax +497614520714 & Accessible online at: \\
$\begin{array}{l}\text { E-mail Information@Karger.de } \\
\text { www.karger.com }\end{array}$ & www.karger.com/tmh
\end{tabular}




\section{Gültigkeitsbereich}

Die oben genannten Richtlinien sowie die nachfolgend im Text aufgeführten Verordnungen und Gesetze für die Herstellung von Blut- und Blutbestandteilkonzentraten gelten für alle Einrichtungen, in denen präparative Hämapheresen durchgeführt werden. Die präparative Hämapherese stellt zusätzliche Anforderungen an die Organisation sowie die personelle, technische und räumliche Ausstattung der Zentren. Die vorliegenden Empfehlungen beziehen sich nicht auf die Durchführung therapeutischer Hämapheresen zur direkten Behandlung von Patienten.

\section{Organisation und Ausstattung der Zentren}

\section{Arzneimittelrecht}

Die bei der präparativen Hämapherese mit Zellseparatoren gewonnenen Blutbestandteilkonserven sind Arzneimittel. Ihre Herstellung ist an eine Herstellungserlaubnis durch die zuständige Behörde im Sinne des Arzneimittelgesetzes (AMG) gebunden. Die Zentren, die Blutzellseparatoren betreiben, unterliegen deshalb den Vorschriften des jeweils gültigen AMG [7].

\section{Räumliche Voraussetzungen}

Herstellungsräume müssen GMP-gerecht (GMP = good manufacturing practice; gute Herstellungspraxis) ausgestattet und eingerichtet sein [11]. Die Hämaphereseeinheit muss für ein Notarztteam in ausreichender Zeit erreichbar sein, wenn nicht ein in der Notfallmedizin ausreichend ausgebildeter und erfahrener Arzt in der Zellseparatoreinheit anwesend ist. Der Spender muss jederzeit von allen Seiten zugänglich sein, hier$\mathrm{zu}$ ist ein Raumbedarf pro Separationsplatz von mindestens $10 \mathrm{~m}^{2} \mathrm{zu}$ veranschlagen.

\section{Notfallmedizinische Ausstattung}

Die Hämaphereseeinheit muss notfallmedizinisch ausgerüstet sein. Der Notfallplan und die entsprechende Ausrüstung sind mit dem zuständigen Notarzt abzustimmen. Der Notfallplan sollte organisatorisch eingeübt sein und in regelmäßigen Abständen trainiert werden. Das an der Hämapherese beteiligte Personal muss notfallmedizinische Kenntnisse besitzen und sich regelmäßig fortbilden.

\section{Ärztliches Personal}

Die Hämapherese muss unter ärztlicher Aufsicht und Weisung erfolgen.

Der Leiter der Einrichtung, in der Hämapheresen durchgeführt werden, muss gemäß Abschnitt 1.4.2.1 der Hämotherapierichtlinien [1] Facharzt für Transfusionsmedizin sein. Der für die Hämaphereseeinheit verantwortliche Arzt muss ausreichende Erfahrungen in der Notfallmedizin [1] und Erfahrung mit extrakorporalen Systemen erworben haben. Eine zusätzliche Ausbildung am Zellseparator des jeweils eingesetzten Typs ist notwendig und zu dokumentieren.
Ärzte, die am Zellseparator selbständig tätig werden, sollten neben notfallmedizinischen Kenntnissen praktische Erfahrungen mit mindestens 20 Hämapheresen je Separationssystem haben. Zusätzlich ist die Teilnahme an einem von der DGTI anerkannten Seminar über Hämapherese erforderlich, die alle 3 Jahre wiederholt werden sollte.

\section{Nichtärztliches Personal}

Die unmittelbare Überwachung und Durchführung von Zellseparationen erfordert eine qualifizierte Fachkraft als Operator, die als Funktionsstelle im Stellenplan ausgewiesen sein sollte. Bei Hämapheresen sollte ein qualifizierter Operator gleichzeitig nicht mehr als 3 Zytapheresen oder 5 Plasmapheresen überwachen.

Der für die Hämapherese verantwortliche Operator sollte Kenntnisse in der Notfallmedizin besitzen und 2 Monate an extrakorporalen Systemen ausgebildet sein. Die Hämaphereseausbildung sollte durch die regelmäßige Teilnahme an von der DGTI anerkannten Hämaphereseseminaren ergänzt werden. Alle Pflegekräfte sollten unter Anleitung über mindestens 3 Monate intensive Erfahrungen in der Hämaphereseeinheit gesammelt haben und regelmäßig Hämapheresen betreuen. Die Anzahl der in einer Hämaphereseeinheit einzusetzenden Operatoren richtet sich nach der Hämapheresefrequenz, um für den einzelnen Operator eine ausreichende Routine sicherzustellen. Hierzu sind monatlich zumindest 5 Hämapheresen je Operator erforderlich.

Insbesondere muss das Personal durch eine ausführliche Einweisung am Zellseparator in der Lage sein, alle Störungen rasch zu erkennen und entsprechend seinem Aufgaben- bzw. Verantwortungsbereich zu beheben.

\section{Ausbildungspersonal}

Die Ersteinweisung am Zellseparator durch Ausbilder des Herstellers erfordert neben technisch-apparativen auch medizinische Kenntnisse. Die Ausbilder sollten daher die Voraussetzungen erfüllen, die auch an Operatoren gestellt werden. Ein Katalog der erforderlichen technisch-apparativen und medizinischen Kenntnisse sollte durch die Sektion «Präparative und therapeutische Hämapherese» der DGTI in Abstimmung mit den Geräteherstellern erstellt werden. Die Ausbilder dokumentieren die Ersteinweisung der Ärzte und Operatoren im Medizinproduktebuch und stellen bei erfolgreicher Geräteeinweisung ein Zertifikat aus.

\section{Zellseparatoren}

Die Zellseparatoren müssen im Hinblick auf die Spendersicherheit vollautomatisch arbeiten und mit selbstkontrollierenden Überwachungssystemen ausgerüstet sein. Zellseparatoren müssen den Vorschriften des Medizinproduktegesetzes (MPG) entsprechen $[1,12]$ und gemäß den Vorschriften der Medizinproduktebetreiberverordnung gehandhabt werden $[1,13]$.

Die Überprüfung der Alarmsysteme und Detektoren muss vor Beginn der Separation erfolgen und darf nicht fakultativ aus- 
schaltbar sein. Überwachungssysteme sollten Zu- und Rückfluss, Unterdruck und Luft im System, Dosierung der Antikoagulanzien, Hämolysen, Undichtigkeiten, Temperatur in der Zentrifuge, Pumpengeschwindigkeit und separiertes Blutvolumen kontrollieren. Der Separationsstatus muss optisch und eventuelle Störfälle müssen zusätzlich akustisch angezeigt und online dokumentiert werden. Bei Gefährdung des Spenders muss das Gerät die Separation automatisch unterbrechen.

Die in den Hämotherapierichtlinien [1] unter Abschnitt 2.6.3 genannten maximalen Bruttoentnahmevolumina (einschließlich Antikoagulanz, zuzüglich Untersuchungsproben) sind zu beachten. Ferner sollte das entnommene Blutvolumen 15\% des zirkulierenden Körperblutvolumens nicht überschreiten. Bei diskontinuierlichen Verfahren sollten das aktuelle extrakorporale Blutvolumen sowie die Anzahl der Sammelzyklen angezeigt werden, um die Spendersicherheit zu gewährleisten. Generell sollten die Verfahren eine kontinuierliche Qualitätskontrolle der Zell/Plasma-Trennung während der Hämapherese ermöglichen.

\section{Einmalsysteme}

Grundsätzlich müssen Einmalsysteme Anwendung finden, die ein sogenanntes «Predonation-Sampling-System» aufweisen. Dem Hersteller obliegt eine besondere Verantwortung für die Sicherheit der Einmalsysteme. Für lagerungsfähige Blutbestandteilpräparate müssen die Einmalsysteme vollständig oder funktionell geschlossen sein [7]. In diesem Sinne gilt das System auch als geschlossen, wenn der Rückgabeschlauch an einer Nadel konnektiert werden muss, vorausgesetzt, dass vom System sichergestellt wird, dass die Richtung des Rückflusses nur zum Spender führt.

\section{Spenderauswahl und -untersuchung}

Der Hämapheresespender muss den in den Hämotherapierichtlinien unter Abschnitt 2.6.4 genannten Anforderungen entsprechen [1], und seine Venenverhältnisse sollten für Hämaphereseverfahren geeignet sein.

Die Einnahme von thrombozytenfunktionseinschränkenden Mitteln muss mindestens 7 Tage, bei Herstellung von Thrombozytenkonzentraten mindestens 10 Tage zurückliegen.

Werden bei Granulozytapheresen Sedimentationsbeschleuniger, Kortikoide und/oder Zytokine appliziert, so ist insbesondere darauf zu achten, ob sich bei Anamnese oder klinischer Untersuchung Hinweise auf spezielle Risiken wie Gastritiden, Ulzera, psychiatrische Vorerkrankungen, Überempfindlichkeiten, Hypertonie oder Diabetes mellitus ergeben. Diese sind vor der Medikamentenapplikation weiter abzuklären. Beim Einsatz von Steroiden sollte z.B. vor Mobilisierung eine Blutzuckerbestimmung durchgeführt werden [1].

Bei Thrombo- und Erythrozytapheresen muss die Thrombozytenzahl des Spenders vor bzw. innerhalb von 15 min nach Beginn der Apherese bestimmt werden und mehr als 150 G/l und weniger als $450 \mathrm{G} / \mathrm{l}$ betragen. Die Leukozytenzahl des Spenders, vor bzw. innerhalb von 15 min nach Beginn der Apherese bestimmt, muss bei Thrombo- und Erythrozytapheresen mehr als $3 \mathrm{G} / 1$ und weniger als $13 \mathrm{G} / \mathrm{l}$ betragen.

Das Verfahren sollte so gewählt werden, dass im Regelfall nach der Thrombozytapherese die Blutplättchenwerte des Spenders nicht unter $100 \mathrm{G} / 1$ abfallen.

Wird bei einer Apherese kein Erythrozytenkonzentrat (EK) gewonnen, soll bei männlichen Spendern der Wert für $\mathrm{Hb}$ $>130 \mathrm{~g} / \mathrm{l}$ und bei weiblichen Spendern $>120 \mathrm{~g} / 1$ sein.

Wird bei einer Spende ein EK gewonnen, soll bei männlichen Spendern der Wert für $\mathrm{Hb}>135 \mathrm{~g} / 1$ und weiblichen Spendern $>125 \mathrm{~g} / \mathrm{l}$ sein. Werden bei einer Spende zwei EKs gewonnen, ist bei allen Spendern ein Wert für $\mathrm{Hb}>140 \mathrm{~g} / \mathrm{l}$ und ein Körpergewicht von mindestens $70 \mathrm{~kg}$ erforderlich [14]. Bei Frauen ist bei Spende von mehr als einem EK pro Jahr und bei Männern bei Spende von mehr als drei EKs pro Jahr die potentielle Entwicklung eines Eisenmangels zu beachten, die eine prophylaktische Eisensubstitution erfordert. Insbesondere bei Spende von zwei EKs sollte deshalb die Spendereignung durch eine Untersuchung des Eisenhaushalts (z.B. Ferritinbestimmung) überprüft werden.

\section{Spenderaufklärung}

Vor der Hämapherese ist der Spender über das Verfahren, die möglichen Nebenwirkungen und die Gefahren durch einen Arzt mündlich und schriftlich aufzuklären. Eine besonders eingehende Aufklärung muss bei Verwendung von Medikamenten (z.B. hämatopoetische Wachstumsfaktoren, Steroide, Sedimentationsbeschleuniger, Heparin) erfolgen. Bei der Anwendung von Medikamenten sind $\S 8$ und 9 des Transfusionsgesetzes [6] zu beachten.

Die Einwilligung des Spenders für die Vorbehandlung und die Hämapherese sowie die erfolgte Aufklärung sind schriftlich zu dokumentieren (Beispiel: Abb. 1).

\section{Versicherungsschutz}

Der Abschluss einer Berufshaftpflichtversicherung durch den Arbeitgeber für das Hämapheresepersonal unter Angabe der Tätigkeitsmerkmale und einer Unfallversicherung für den Zellseparatorspender, die auch eventuelle Spätschäden trägt, ist zu empfehlen.

\section{Durchführung der Hämapheresen}

Die Hämapherese sollte in einer für den Spender bequemen Position erfolgen. Eine sofortige Schocklagerung muss möglich sein.

Die Vorbereitung der Geräte erfolgt durch den Operator. Ein zweiter Operator oder der zuständige Arzt haben anhand einer Prüfliste eine Sicherheitsüberprüfung der Geräte vor Beginn der Hämapherese durchzuführen und zu dokumentieren. Hierbei ist insbesondere die korrekte Lage und Füllung 
Name, Vorname, Geb.-Datum:

Hiermit erkläre ich freiwillig meine Zustimmung zur Spende von Granulozyten ( Leukozyten ) mit Hilfe eines Zellseparators, von Sedimentationsbeschleunigern ( Hydroxyäthylstärke ) und blutgerinnungshemmenden Mitteln ( Citrat ) sowie zur Injektion des Wachstumsfaktors für Granulozyten ( G-CSF ) und / oder Einnahme von Cortison.

Ich wurde von Frau/Herrn Dr.

über den Spendevorgang von Granulozyten/Leukozyten mittels Zellseparation, über die damit notwendige Verwendung von Sedimentationsbeschleunigern ( z.B. Hydroxyäthylstärke ), die Verabreichung des Wachstumsfaktors für Granulozyten (G-CSF) und / oder Cortison, die möglichen Gefahren und Nebenwirkungen vollständig und für mich verständlich informiert. Es ist meine freie Entscheidung, daß mir G-CSF gespritzt wird. Ich habe keine weiteren Fragen mehr.

Spendevorgang

Ich habe verstanden, daß nur 8 - 16 Stunden vor der Granulozytenspende der Wachstumsfaktor für Granulozyten in das Unterhautfettgewebe ( subcutan) gespritzt und / oder Cortison eingenommen werden muß, um die Granulozytenzahl in meinem Blut zu erhöhen. Bei der Zellseparation wird mein Blut aus einer Armvene unter Zusatz von gerinnungshemmenden Mitteln und von Sedimentationsbeschleunigern entnommen und im Zellseparator durch Zentrifugation aufgetrennt. Ein Teil der für die Transfusion benötigten Granulozyten/Leukozyten wird entnommen. Die übrigen Blutbestandteile werden je nach Separatortyp ständig oder mit Unterbrechung über dieselbe oder eine andere Armvene wieder zurückgegeben. Hierbei gelangen auch geringe Mengen an gerinnungshemmenden Mitteln und Sedimentationsbeschleunigern in meinen Kreislauf. Der Spendevorgang kann bis zu 4 Stunden dauern. Über das erforderliche Verhalten vor, während und nach der Spende bin ich informiert worden. Nach der Spende kann ich nur durch den zuständigen Arzt entlassen werden.

\section{Gefahren und Nebenwirkungen}

Über die nicht völlig auszuschließenden Gefahren und Nebenwirkungen bin ich aufgeklärt worden und nehme sie in Kauf. Durch frühzeitige Mitteilung von Mißempfindungen während der Spende meinerseits können Nebenwirkungen vermieden werden. Sollte ein Zwischenfall bei der Zellseparation auftreten, bin ich mit den zur Abwendung gesundheitlicher Schäden notwendigen Maßnahmen einverstanden. Mögliche Nebenwirkungen sind:

a) Wachstumsfaktor für Granulozyten ( G-CSF ): Knochen- u. Kopfschmerzen, Müdigkeit, Erbrechen, Schlafstörungen b) Zellseparation (äußerst selten bzw. nahezu ausgeschlossen): Kreislaufschwäche ( Schweißausbruch, Übelkeit, Erbrechen, Ohnmacht), Zitrat-Reaktionen (Kribbeln an Fingern, Zehen, Mund; Muskelverkrampfungen, unregelmäßiger Herzschlag), Punktion von atypisch verlaufenden Nervenästen (Nervenreizung, -schädigung und -lähmung) und Arterien sowie Luftembolie, Blutverlust Gerinnsel und Blutzerfall.

c) Sedimentationsbeschleuniger ( selten ): Überempfindlichkeitsreaktionen ( Allergie ), Blutungsneigung

d) Cortison: individuelle Unverträglichkeit bei kurzfristiger Anwendung

Ich weiß, daß es sich bei dem Wachstumsfaktor für Granulozyten um ein Medikament handelt, von dem nach dem derzeitigen Wissens stand keine Gefährdung ausgeht, jedoch Langzeiterfahrungen noch nicht vorliegen können.

Eine Kopie der Informationen zur Granulozytenspende und dieser Einverständniserklärung habe ich erhalten.

\section{Datenschutz}

Ich bin darüber informiert worden, daß die erhobenen Daten den Bestimmungen des Datenschutzgesetzes unterliegen. Die Erhebung, Verarbeitung und Nutzung meiner personenbezogenen Daten erfolgt im Rahmen des Transfusions- und Infektionsschutzgesetzes.

Ich fühle mich zur Zeit völlig gesund und habe seit 7 Tagen keine Medikamente eingenommen außer den für die Spende erforderlichen. => Für Frauen: Ich versichere, nicht schwanger zu sein.

Fig. 1. Aufklärung zur Granulozytapherese.
Ort / Datum: $\quad$ Spender:

Operator:

Arzt: des Apheresesystems, die richtige Wahl der Lösungen, insbesondere des Antikoagulanz, sowie die richtige Einstellung bzw. Programmierung der spenderbezogenen und herstellungsrelevanten Parameter des Separationsprogramms zu kontrollieren. Ein aus der Umverpackung entnommenes System darf nur am gleichen Arbeitstag verwendet werden. Zwischen Vorfüllung des Apheresesystems und Beginn der Hämapherese sollten nicht mehr als $4 \mathrm{~h}$ liegen.

Die sachgerechte Durchführung und ständige Überwachung der Zellseparation erfolgt durch den Operator. Der Spender ist regelmäßig nach seinem Befinden, insbesondere nach eventuellen Zeichen der Zitratnebenwirkung, zu befragen. Bei Einschränkung des Wohlbefindens sowie bei technischen Störungen ist die Zellseparation zu unterbrechen und der verantwortliche Arzt zu rufen. Über gegebenenfalls einzuleitende Maßnahmen, Abbruch des Verfahrens oder Fortsetzung der Zellseparation und Verwendbarkeit des Präparats entscheidet der verantwortliche Arzt.

Zumindest am Beginn der Zellseparation sowie bei technischen und medizinischen Störungen ist die unmittelbare 
Anwesenheit des verantwortlichen Arztes erforderlich. Für die übrige Zeit der Zellseparation muss der verantwortliche Arzt jederzeit herbeigerufen werden können.

Alle aufgeführten Kontrollen sowie Störungen bzw. Änderung des Programms sind zu protokollieren.

Die Entlassung erfolgt durch den zuständigen Arzt bzw. einen von ihm bevollmächtigten Mitarbeiter. Dabei muss sichergestellt sein, dass sich der Spender wohlfühlt, kreislaufstabil ist und die Punktionsstellen richtig versorgt sind. Es wird empfohlen, dem Spender eine ärztliche Mitteilung über die Art der Spende und eventuell applizierte Medikamente (z.B. Eintrag im Spenderausweis) auszustellen.

Über jede Apherese sowie über eventuell anschließende Präparationen ist ein Protokoll anzufertigen, das die Angaben über das Verfahren sowie Art, Menge und Chargenbezeichnung der verwendeten Materialien und gegebenenfalls die Ergebnisse der Qualitätskontrollen enthält.

\section{Präparation von Thrombozytapheresekonzentraten}

Ziel ist die Gewinnung von therapeutischen Einheiten, die im Mittel $3 \times 10^{11}$ und stets mehr als $2 \times 10^{11}$ Thrombozyten in einem Volumen von 200-300 ml und weniger als $1 \times 10^{6}$ Leukozyten und weniger als $1 \times 10^{9}$ Erythrozyten je Einheit enthalten.

Eine zusätzliche Sammlung weiterer Komponenten ist bei Verwendung eines funktionell geschlossenen Systems unter Beachtung der Grenzwerte für die jeweiligen Komponenten möglich.

\section{Präparation von Erythrozytapheresekonzentraten}

Die durch Apherese gewonnene therapeutische Einheit entspricht einem leukozytendepletierten EK in Additivlösung mit einem Volumen von 300-350 ml, einem Hämatokrit von 0,5-0,7 1/1, einem Mindesthämoglobingehalt von $40 \mathrm{~g}$ und einem Restleukozytengehalt von weniger als $1 \times 10^{6} /$ Einheit.

\section{Präparation von Plasma}

Die therapeutische Einheit des Produkts «gefrorenes Frischplasma» enthält 200-300 ml antikoaguliertes Plasma mit einem Restzellgehalt von $<50 \times 10^{9} / 1$ Thrombozyten und $<6 \times$ $10^{9} / 1$ Erythrozyten und $<1 \times 10^{6}$ Leukozyten je Einheit.

\section{Multikomponentenspenden}

Die Multikomponentenspende umfasst die Präparation von 2 oder mehr der oben genannten Komponenten. Für jede der Einzelkomponenten gelten die jeweils genannten Qualitätskriterien und Grenzwerte. Ferner ist zu beachten, dass bei der Sammlung mehrerer Komponenten das entnommene Gesamtvolumen $750 \mathrm{ml}$ (inklusive Antikoagulanz), respektive 15\% des zirkulierenden Körperblutvolumens nicht überschreitet.

\section{Präparation von Granulozytapheresekonzentraten}

Die angestrebte therapeutische Dosis pro Transfusion beträgt mindestens $1 \times 10^{10}$ Granulozyten $/ \mathrm{m}^{2}$ Körperoberfläche des
Empfängers. Der Hämatokritwert des Produkts sollte maximal 0,30 1/l betragen. Allogene Granulozytenpräparate sind aufgrund des hohen Risikos einer Graft-versus-Host-Reaktion grundsätzlich mit $30 \mathrm{~Gy}$ zu bestrahlen.

Die Gewinnung von Granulozyten mit Zellseparatoren ist unter Verwendung von Sedimentationsbeschleunigern in therapeutisch wirksamen Dosen möglich. Als Sedimentationsbeschleuniger sollte 6\%ige hochmolekulare Hydroxyethylstärke (HES, MW 450) eingesetzt werden. Pro Granulozytapherese sollten maximal $750 \mathrm{ml}$ HES verwendet werden. Durch Sedimentationsbeschleuniger kann es zu Unverträglichkeitsreaktionen kommen. Die sehr niedrige Frequenz von anaphylaktoiden Reaktionen bei entsprechender Vorsorge berechtigt zu ihrem Einsatz. Bei Verwendung von HES sollte eine biologische Vortestung (z.B. $1 \mathrm{ml}$ i.v. mit zirka $5 \mathrm{~min}$ Reaktionszeit) durchgeführt werden.

Zur Antikoagulation hat sich eine Mischung von $50 \mathrm{ml}$ einer $37,5 \%$ igen Natriumzitratlösung in $500 \mathrm{ml}$ HES bewährt. Zur Verringerung möglicher Zitratreaktionen kann der Zitratanteil weiter reduziert werden, wenn der Antikoagulationslösung Heparin zugesetzt wird. Gemische von Antikoagulanzien und Sedimentationsbeschleunigern sollten wegen der Gefahr der Entmischung viertelstündig durchmischt werden. Für die Konditionierung von Spendern mit dem Ziel der Steigerung der peripher zirkulierenden Gesamtgranulozytenzahl und folglich der Sammeleffizienz bei der Granulozytapherese stehen als Stimulantien G-CSF (z.B. $5 \mu \mathrm{g} / \mathrm{kg} \mathrm{KG)} \mathrm{und} \mathrm{Korti-}$ koide (z.B. 8-12 mg Dexamethason oder 80-120 mg Prednisolon, jeweils auf 1-2 Applikationen verteilt) allein oder in Kombination zur Verfügung.

\section{Präparation von Lymphozytapheresekonzentraten}

Lymphozytenkonzentrate können zur adoptiven Immuntherapie verwendet werden. Die Präparation erfolgt von nichtstimulierten Spendern analog der Herstellung von Blutstammzellprodukten. Die anzustrebende therapeutische Zellzahl ist vm individuellen Bedarf des Patienten abhängig und kann bis $1-2 \times 10^{8} / \mathrm{kg} \mathrm{KG}$ des Empfängers betragen. Um diese Zellzahl zu sammeln, muss in der Regel das zwei- bis dreifache Zirkulationsvolumen prozessiert werden.

\section{Präparation von Monozytapheresekonzentraten}

Monozytenkonzentrate können z.B. als Ausgangsmaterial zur Generierung dendritischer Zellen für die Tumortherapie verwendet werden. Die Präparation erfolgt von nichtstimulierten Spendern analog der Herstellung von Blutstammzellprodukten. Die anzustrebende Zellzahl beträgt $1-5 \times 10^{9}$ Monozyten und kann in der Regel mit einem Blutdurchflussvolumen vom anderthalb- bis dreifachen Zirkulationsvolumen erzielt werden.

\section{Praparation von Blutstammzellkonzentraten}

Blutstammzellkonzentrate können zur Regeneration des blutbildenden Systems nach myelosuppressiver Therape eingesetzt werden. Die individuell benötigte Zellzahl wird mit dem 
Transplantationszentrum patientenbezogen abgestimmt, wobei gemäß den Abschnitten 2.6.4.6.4 und 2.8.7 der Hämotherapierichtlinien die anzustrebende Mindestdosis von $4 \times 10^{6} \mathrm{CD} 34+$ Zellen/kg KG für die allogene und $2 \times 10^{6} \mathrm{CD} 34+$ Zellen $/ \mathrm{kg}$ $\mathrm{KG}$ für die autologe Stammzelltransplantation berücksichtigt werden sollte.

Für die Stammzellmobilisierung wird derzeit überwiegend G-CSF verwendet. Vor der Mobilisierung mit Wachstumsfaktoren hat eine Eignungsuntersuchung der Spender bzw. Patienten zu erfolgen. Im Rahmen dieser Voruntersuchung ist auch zu entscheiden, ob ein peripher- oder ein zentralvenöser Zugang für die Stammzellseparation notwendig ist.

Bei Verwendung zentraler Venenkatheter ist eine entsprechende Aufklärung und Erfahrung des Operators im Umgang mit diesem Katheter Voraussetzung, das erhöhte Komplikationsrisiko ist zu berücksichtigen, und der Eingriff zu dokumentieren. Die Eignung der Spender und Patienten zur Blutstammzellmobilisation mit Wachstumsfaktoren und zur Apherese wird von dem für die Zytapherese zuständigen Arzt durch Anamnese und Untersuchung festgestellt.

Als Antikoagulans wird ACD im geeigneten Mischungsverhältnis empfohlen. Bei zusätzlicher Antikoagulation mit Heparin (zirka $15 \mathrm{IE} / \mathrm{kg} \mathrm{KG/h}$ ) kann die Zitratzufuhr auf bis zu 50\% reduziert werden [15].

Das prozessierte Blutvolumen soll in der Regel pro Apherese das Vierfache des jeweiligen Körperblutvolumens nicht übersteigen. Eine Apheresesitzung sollte auf maximal $5 \mathrm{~h}$ begrenzt werden.

Werden zur Stammzellapherese homologe Blutprodukte benötigt (z.B. zum Vorfüllen des Apheresesets mit Erythrozyten bei kleinen Kindern oder zur Behandlung einer Thrombozytopenie), sind diese Präparate mit $30 \mathrm{~Gy}$ zu bestrahlen. Bei Patienten sollte der Thrombozytenwert vor der Stammzellapherese über $30 \mathrm{G} / \mathrm{l}$ liegen. Sind die Thrombozytenwerte nach der Apherese unter $30 \mathrm{G} / 1$, ist eine entsprechende Überwachung des Patienten zu gewährleisten. Die Indikation zur Thrombozytensubstitution wie z.B. die Retransfusion der mit der Stammzellapherese entnommenen autologen Thrombozyten ist individuell zu prüfen.

Bei Spendern sollte zur Entlassung der Thrombozytenwert über $80 \mathrm{G} / 1$ liegen. Gegebenenfalls ist eine Retransfusion der mit der Stammzellapherese entnommenen autologen Thrombozyten durchzuführen.

\section{Unterbrechung der Hämapherese}

Bei mikroprozessorgesteuerten Systemen kann jede Abweichung ein Hinweis auf eine weitergehende technische Störung sein und muss abgeklärt werden. Daher ist bei allen nicht sofort behebbaren technischen Problemen und Störungen des Wohlbefindens des Spenders die Hämapherese sofort zu unterbrechen. Die Zugänge sind offenzuhalten und der verantwortliche Arzt ist herbeizurufen. Die Hämapherese ist nach Maßgabe des verantwortlichen Arztes vorzeitig zu beenden, wenn medizinische Gründe (z.B. Arrhythmien, Kreislaufkollaps, Krämpfe, Auftreten von Exanthemen usw.) oder nicht behebbare bzw. nicht erklärbare technische Störungen vorliegen. Bei schwerwiegenden medizinischen Zwischenfällen ist der Notfallplan anzuwenden.

Nach Auftreten nicht behebbarer gerätetechnischer Probleme mit Zellseparatoren ist vor neuerlichem Einsatz eine Überprüfung durch den zuständigen technischen Wartungsdienst notwendig. Nach jeder Reparatur oder Wartung sind die Zellseparatoren durch die verantwortliche Person für den Betrieb freizugeben.

\section{Spendefrequenz}

Die Spendefrequenz am Zellseparator ist so zu wählen, dass keine gesundheitliche Schädigung des Spenders zu erwarten ist. Insbesondere darf die Thrombozytenentnahme zu keinem Blutungsrisiko vonseiten der Thrombozytenzahl und -funktion oder zu einer überschießenden Regeneration führen. Bei Erythrozytenverlust im Rahmen von Zellseparationen (vor allem bei Granulozytapheresen) ist zu beachten, dass die entnommenen Erythrozyten einen relativ hohen Prozentsatz an Retikulozyten enthalten.

\section{Thrombozytapherese}

Unter regelmäßiger Überwachung sind bis zu 26 Apheresen mit bis zu jeweils $6 \times 10^{11}$ Thrombozyten/Jahr möglich. Innerhalb von 5 Tagen sind tägliche Thrombozytapheresen mit bis zu jeweils $3 \times 10^{11}$ Thrombozyten ohne gleichzeitige Plasmapherese vertretbar. Nach einem 5-Tage-Zyklus ist bis zur nächsten Einzelspende ein Abstand von 14 Tagen einzuhalten. Ein erneuter 5-Tage-Zyklus ist unter besonderer Beachtung der Thrombozytenwerte frühestens nach 3 Monaten möglich. Der zeitliche Abstand zwischen zwei Doppelkonzentrat-Thrombozytapheresen sollte mindestens 14 Tage, bei höheren Zellmengen mindestens 21 Tage betragen. Werden pro Hämapherese mehr Zellen separiert als für das Standardverfahren aufgeführt, ist die Spendefrequenz entsprechend herabzusetzen.

\section{Erythrozytapherese}

Pro Jahr ist die Entnahme von bis zu $1000 \mathrm{ml}$ Erythrozyten (entspricht 4 EKs) bei Frauen und von bis zu 1500 ml (entspricht 6 EKs) bei Männern möglich. Der zeitliche Abstand zwischen zwei Erythrozytapheresen mit Entnahme eines EK soll bei Frauen 12 Wochen und bei Männern 8 Wochen nicht unterschreiten. Nach Erythrozytapheresen mit Entnahme von zwei EKs soll der Abstand zur nächsten Erythrozytenspende bei Frauen 26 Wochen und bei Männern 18 Wochen nicht unterschreiten. Insbesondere bei Spende von zwei EKs in einer Apherese sollte die Spendereignung durch eine Untersuchung des Eisenhaushalts (z.B. Ferritinbestimmung) überprüft werden. 
Tab. 1. Mindestabstände bis zur Spende von Blutprodukten nach allogener Stammzellapherese

\begin{tabular}{ll}
\hline Spenden für andere Patienten & $\begin{array}{l}\text { Abstand zur } \\
\text { Stammzellspende }\end{array}$ \\
\hline Vollblut & 3 Monate \\
Erythrozytapherese & 6 Monate \\
Leukozytapherese & 6 Monate \\
Thrombozytapherese & 6 Monate \\
Multikomponentenspende & 6 Monate \\
Plasmapherese & 6 Monate \\
Lymphozytapherese & 2 Jahre \\
Granulozytapherese mit G-CSF-Stimulation & 2 Jahre \\
Blutstammzellapherese mit G-CSF-Mobilisation & 2 Jahre \\
\hline
\end{tabular}

\section{Granulozytapherese}

Infolge der Verwendung von Sedimentationsbeschleunigern ist die Frequenz auf maximal 4 Granulozytapheresen/Spender/Jahr limitiert, die auch an aufeinanderfolgenden Tagen durchgeführt werden können. Nach einer Granulozytapherese ist bis zur nächsten Erythrozyten- oder Vollblutspende ein Abstand von mindestens 8 Wochen, nach 2 und mehr Granulozytapheresen von mindestens 16 Wochen einzuhalten.

\section{Lympho- und Monozytapherese}

Die Anzahl der Lympho- oder Monozytenspende soll 6 Apheresen/Jahr nicht überschreiten. Wird bei einer Apherese weniger als das Zweifache des Zirkulationsvolumens prozessiert, kann die Spendefrequenz entsprechend angehoben werden. Wird bei einer Apherese mehr als das Zweifache des Zirkulationsvolumens prozessiert, sollte die Spendefrequenz entsprechend reduziert werden. Der Abstand zu einer weiteren Hämapherese (mit Ausnahme der Stammzellapherese) sollte 14 Tage nicht unterschreiten.

\section{Stammzellapherese}

Nach allogener Blutstammzellapherese sollten die in Tabelle 1 aufgelisteten Mindestabstände bis zur Spende von Blutprodukten für andere Patienten als den Transplantatempfänger nicht unterschritten werden.

\section{Andere Hämapheresen}

Bezüglich der Frequenzen für die übrigen Hämapheresearten wird auf die Hämotherapierichtlinien [1] Abschnitt 2.6.3 «Häufigkeit und Menge der Hämapheresespenden» verwiesen.

\section{Qualitätskontrolle und -sicherung}

Außer den in den Empfehlungen des Europarats [16] und in den Hämotherapierichtlinien [1] aufgestellten Minimalanforderungen sollte - analog zu den AABB-Standards (5.7.5.16) besonders bei Thrombo- und allen Leukozytapheresekonzen- traten der Gehalt der wirksamen Bestandteile und der kontaminierenden Zellen in jedem Präparat kontrolliert und nur Präparate ausgegeben werden, die die Spezifikationen erfüllen [17].

Nach einem unbefriedigenden Transfusionsergebnis durch ein Thrombozytapheresekonzentrat ist die Verträglichkeit und der Erfolg weiterer Thrombozytentransfusionen schriftlich in Form eines Transfusionsberichts an die transfusionsmedizinische Einrichtung zu melden, da davon die weitere Indikationsstellung und Auswahl der Spender abhängig sein kann. Dies gilt insbesondere für die Transfusion von Leukozytapheresepräparaten oder HLA-kompatiblen Thrombozytapheresepräparaten. Der therapeutische Effekt von Granulozyten muss jedoch nicht zwangsläufig mit dem posttransfusionellen Granulozytenanstieg korrelieren.

\section{Maßnahmen zur Qualitätssicherung und Verbesserung der Verfahren}

Alle transfusionsmedizinischen Einrichtungen sollten Probleme, Störungen und Nebenwirkungen bei Zellseparationen regelmäßig dem Obmann der Sektion «Präparative und therapeutische Hämapherese» der DGTI berichten. Die Hersteller und Vertriebsorganisationen von Zellseparatoren und Zellseparatorsystemen sind verpflichtet, Probleme der Technik oder des Materials zu melden.

\section{Spendersicherheit}

Oberstes Gebot bei der Durchführung präparativer Hämapheresen ist die Spendersicherheit, die durch eine sachkundige, ärztliche Eignungsuntersuchung vor jeder Apherese und entsprechende Überwachung während der Apherese gewährleistet sein muss. Neben einer umfassenden Aufklärung und schriftlichen Einwilligung sind die Freiwilligkeit, die Häufigkeit und die Menge der Spenden wesentliche Punkte, die zu beachten sind. Die Übermittlung relevanter Befunde und die gegebenenfalls erforderliche Nachbetreuung der Spender müssen sichergestellt sein. Gemäß $§ 31$ der Arzneimittel- und Wirkstoffherstellungsverordnung [18] müssen in allen Spendeeinrichtungen ein Qualitätsmanagementsystem etabliert und alle kritischen Arbeitsabläufe in Standardarbeitsanweisungen festgelegt sein. Der Spendebereich muss die sichere Entnahme sowie eine möglicherweise erforderliche Behandlung der spendenden Person ermöglichen. Im Rahmen der Dokumentations- und Meldepflicht sind schwerwiegende unerwünschte Reaktionen des Spenders bei der Gewinnung von Blutzubereitungen anzuzeigen. Die Aphereseeinrichtungen sollten deshalb im Rahmen der Qualitätssicherung geeignete Dokumentations- und Meldesysteme vorhalten und zur Überprüfung der Arbeitsabläufe Akkreditierungs- und Zertifizierungsverfahren durchführen. 


\section{Literatur}

1 Wissenschaftlicher Beirat der Bundesärztekammer und Paul-Ehrlich-Institut: Richtlinien zur Gewinnung von Blut und Blutbestandteilen und zur Anwendung von Blutprodukten (Hämotherapie). Köln, Deutscher Ärzte-Verlag, 2005.

2 Hämapheresekommission der Deutschen Gesellschaft für Transfusionsmedizin und Immunhämatologie: Durchführung apparativer Plasmapheresen zur Gewinnung von Spenderplasma. Empfehlungen der Ständigen Hämapheresekommission der Deutschen Gesellschaft für Transfusionsmedizin und Immunhämatologie. Infusionsther Transfusionsmed 1994;21:349-354.

3 Burkhardt T, Kadar JG, Matthes G, et al: Durchführung präparativer zellulärer Hämapheresen zur Gewinnung von Blutbestandteilkonzentraten. II. Empfehlungen zur präparativen Leuko- und Thrombozytapherese der Deutschen Gesellschaft für Transfusionsmedizin und Immunhämatologie (DGTI). Infusionsther Transfusionsmed 1998;25: 376-382.

4 Cassens U, Kadar J, Moog R, et al: Durchführung präparativer zellulärer Hämapheresen zur Gewinnung von Blutbestandteilkonzentraten. I. Empfehlungen zur Blutstammzellapherese der Deutschen Gesellschaft für Transfusionsmedizin und Immunhämatologie (DGTI). Infusionsther Transfusionsmed 1998;25:325-335.

5 Deutscher Bundestag: Gesetz zur Regelung des Transfusionswesens (Transfusionsgesetz). BGBl. 1998;I:1752-1760.
6 Deutscher Bundestag: Erstes Gesetz zur Änderung des Transfusionsgesetzes und arzneimittelrechtlicher Vorschriften. BGBl. 2005;I:234-238.

7 Deutscher Bundestag: Zwölftes Gesetz zur Änderung des Arzneimittelgesetzes vom 30. Juli 2004. BGBl. 2004;I:2031-2053.

8 Kommission der Europäischen Gemeinschaft: Richtlinie 2004/23/EG zur Festlegung von Qualitäts- und Sicherheitsstandards für die Spende, Beschaffung, Testung, Verarbeitung, Konservierung, Lagerung und Verteilung von menschlichen Geweben und Zellen. Amtsblatt der Europäischen Union 2004;L102:48-58.

9 Kommission der Europäischen Gemeinschaft: Richtlinie 2002/98/EG zur Festlegung von Qualitäts- und Sicherheitsstandards für die Gewinnung, Testung, Verarbeitung, Lagerung und Verteilung von menschlichem Blut und Blutbestandteilen und zur Änderung der Richtlinie 2001/83/EG. Amtsblatt der Europäischen Union 2003;L33:30-40.

10 Wissenschaftlicher Beirat der Bundesärztekammer und Paul-Ehrlich-Institut: Richtlinien zur Transplantation peripherer Blutstammzellen. Dtsch Ärztebl 1997;94A:1584-1592.

11 Kommission der Europäischen Gemeinschaft: Richtlinie zur Festlegung der Grundsätze und Leitlinien der Guten Herstellungspraxis für Humanarzneimittel und für zur Anwendung beim Menschen bestimmte Prüfpräparate. Amtsblatt der Europäischen Union 2003;L262:22-26.
12 Deutscher Bundestag: Gesetz über die Medizinprodukte (Medizinproduktegesetz - MPG) vom 2.8.1994, BGBI. 1994;1:1963-1984, in der Neufassung durch Bekanntmachung vom 7.8.2002 (BGBl. 1:3146), geändert am 25.11.2003 (BGBl 1:2304). BGBl. 2003.

13 Deutscher Bundestag: Verordnung über das Errichten, Betreiben und Anwenden von Medizinprodukten (Medizinprodukte-BetreiberverordnungMPBetreibV) in der Fassung der Bekanntmachung vom 21.8.2002 (BGBl 1:3396). BGBl. 2002;I:3396.

14 Shi PA, Ness PM: Two-unit red cell apheresis and its potential advantages over traditional wholeblood donation. Transfusion 1999;39:218-225.

15 Malachowski ME, Comenzo RL, Hillyer CD, et al: Large-volume leukapheresis for peripheral blood stem cell collection in patients with hematologic malignancies. Transfusion 1992;32:732-735.

16 Council of Europe: Guide to the Preparation, Use and Quality Assurance of Blood Components, 13 ed. Strasbourg, Council of Europe Publishing, 2007.

17 AABB: Standards for Blood Banks and Transfusion Services, 22 ed. Bethesda, AABB, 2003.

18 Verordnung über die Anwendung der guten Herstellungspraxis bei der Herstellung von Arzneimitteln und Wirkstoffen und über die Anwendung der guten fachlichen Praxis bei der Herstellung von Produkten menschlicher Herkunft (Arzneimittelund Wirkstoffherstellungsverordnung - AMWHV) vom 3. November 2006. BGBl. I S. 2523-2542. 